\title{
Effects of Additives on Coke Reactivity and Sulfur Transformation during Co-pyrolysis of Long Flame Coal and High-Sulfur Coking
}

\section{Coal}

\author{
Wenguang $\mathrm{Li}^{1,2}$, Yanfeng Shen ${ }^{1,2}$, Jiang Guo ${ }^{1,2}$, Jiao Kong1, ${ }^{1,}$, Meijun Wang1,2*, \\ Liping Chang ${ }^{1,2 *}$
}

1 State Key Laboratory of Clean and Efficient Coal Utilization, Taiyuan University of Technology, Taiyuan 030024, P.R China

2 Key Laboratory of Coal Science and Technology (Taiyuan University of Technology), Ministry of Education, Taiyuan 030024, P.R China

\section{List of Figure Captions}

Figure S1. Coke yields of coal blends with the different proportions of GL.

Figure S2. TG and DTG curves of samples.

* Corresponding author. Tel.: +86-0351-6010482

E-mail address: wangmeijun@tyut.edu.cn; lpchang@tyut.edu.cn 




Figure S1. Coke yields of coal blends with the different proportions of GL.
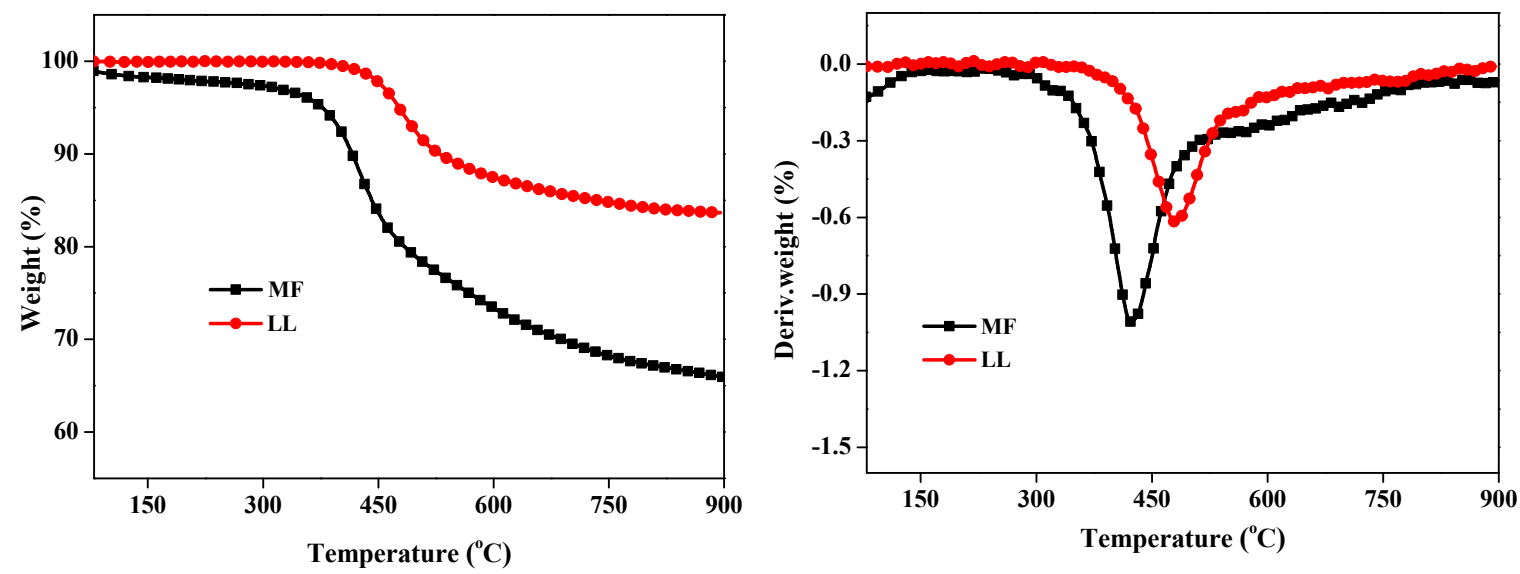

Figure S2. TG and DTG curves of samples. 